\title{
One-pot synthesis of high-capacity silicon-lithium anodes via on- copper growth of a semi-conducting, porous polymer
}

\author{
Jieyang Huang ${ }^{[a]}$, Andréa Martin ${ }^{[a]}$, Anna Urbanski ${ }^{[b]}$, Ranjit Kulkarni ${ }^{[a]}$, Patrick Amsalem ${ }^{[c]}$, Moritz \\ Exner $^{[a]}$, Johannes Müller ${ }^{[c]}$, David Burmeister ${ }^{[a]}$, Norbert Koch ${ }^{[c],[d]}$, Nicola Pinna ${ }^{[a]}$, Petra Uhlmann ${ }^{[b]}$, \\ Michael J. Bojdys ${ }^{*[a],[e]}$
}

[a] Dr. J. Huang, Dr. A. Martin, Dr. R. Kulkarni, M. Exner, D. Burmeister, Prof. Dr. N. Pinna, Prof. Dr. M. J. Bojdys Institut für Chemie and IRIS Adlershof

Humboldt-Universität zu Berlin

Brook-Taylor-Str. 2, 12489 Berlin, Germany

E-mail:m.j.bojdys.02@cantab.net

[b] Dr. A. Urbanski, Prof. Dr. P. Uhlmann

Leibniz-Institut für Polymerforschung Dresden (IPF) e. V

Institut für Physikalische Chemie and Physik der Polymere

01069 Dresden, Germany

[c] Dr. P. Amsalem, J. Müller, Prof. Dr. N. Koch

Institut für Physik and IRIS Adlershof

Humboldt-Universität zu Berlin

Brook-Taylor-Str. 2, 12489 Berlin, Germany

[d] Prof. Dr. N. Koch

Helmholtz-Zentrum Berlin GmbH

Albert-Einstein-Str. 15, 12489 Berlin

[e] Prof. Dr. M. J. Bojdys

Department of Chemistry

King's College London

Britannia House Guy's Campus, 7 Trinity Street, London, SE1 1DB, UK

Supporting information for this article is given via a link at the end of the document.

\begin{abstract}
Silicon-based anodes with lithium ions as charge carriers have the highest predicted charge density of $3579 \mathrm{~mA} \mathrm{~h} \mathrm{~g}^{-1}$ (for $\mathrm{Li}_{15} \mathrm{Si}_{4}$ ). Contemporary electrodes do not achieve this theoretical value largely because conventional production paradigms rely on the mixing of weakly coordinated components. In this paper, a semi-conductive triazine-based graphdiyne polymer network is grown around silicon nanoparticles directly on the current collector, a copper sheet. The porous, semi-conducting organic framework (i) adheres to the current collector on which it grows via cooperative van der Waals interactions, (ii) acts effectively as conductor for electrical charges and binder of silicon nanoparticles via conjugated, covalent bonds, and (iii) enables selective transport of electrolyte and Li-ions through pores of defined size. The resulting anode shows extraordinarily high capacity at the theoretical limit of fully lithiated silicon. Finally, we combine our anodes in proof-of-concept battery assemblies using a conventional cathode, NCM811.
\end{abstract}

\section{Introduction}

Cheap, high-performance, and safe energy storage solutions are needed to address the increasing demand for portable electronics and the transition to electric mobility. Lithium-ion batteries have replaced conventional secondary battery technology (like nickelcadmium and nickel-metal hydride batteries) due to their high energy densities, high operating voltages, and stable capacity retention during open-circuit rest. ${ }^{[1]}$ Lithium metal anodes were quickly replaced by lithium-intercalated graphitic materials in order to avoid the formation of dendrites that resulted in short circuiting of the two electrodes. However, while lithium-graphite intercalates are safer and more stable they have only a tenth of the energy density of metallic lithium. ${ }^{[2]}$ Silicon is a good active material for $\mathrm{Li}$-ion anodes because it has a superior theoretical capacity of $3579 \mathrm{~mA} \mathrm{~h} \mathrm{~g}^{-1}$ (or $8340 \mathrm{~mA} \mathrm{~h} \mathrm{~cm}^{-3}$ ) for $\mathrm{Li}_{15} \mathrm{Si}_{4}$, and unlike some transition metals - it is not toxic and abundant. Moreover, its alloying reaction with lithium triggered at $0.3 \mathrm{~V}$ vs. $\mathrm{Li} / \mathrm{Li}^{+}$, prevents the formation of metallic lithium around the anode during discharging - a detrimental process observed for $\mathrm{Li}$ graphite batteries known as "lithium plating" - and allows the use of Si-Li electrodes under harsher conditions. ${ }^{[3]}$ The large number of lithium atoms that silicon can store, however, induces large volume changes during charge/discharge cycles $(>300 \%) .{ }^{[3]}$ The mechanical stress induced by these drastic volume changes leads to the pulverization of the silicon active material, loss of contact of the electrode film with the current collector, and loss of overall mechanical integrity of the whole electrode. Repeated cycles of expansion and contraction of silicon expose pristine silicon surfaces and induce the reformation of solid electrolyte interfaces (SEI). This process contributes to the gradual consumption of lithium and electrolyte, and it limits the diffusion of charge carriers through the expanding SEI. ${ }^{[3-4]}$ It is difficult to counter these detrimental mechanical and chemical changes to silicon-based electrodes because conventional methods of electrode assembly rely on the mixing of multiple components that are held together by weak, dispersive forces.

In laboratory settings, some strategies were developed to address the inherent flaws of these multi-component assemblies. For example, shaping silicon into hierarchical, nano-sized, or porous structures buffers some of its dramatic volume expansion during lithiation. ${ }^{[4 d]}$ On the downside, nanostructured silicon is susceptible to restructuring during battery operation, and its larger specific surface promotes reactions with the electrolyte to form more of electrochemically inactive SEI. In other approaches silicon particles are encapsulated in a carboneous matrix, ${ }^{[5]}$ or 
they are coated with metal oxides. ${ }^{[6]}$ However, encapsulation of silicon requires supplementary components that do not meaningfully contribute to the volumetric capacity of the electrode and might necessitate the addition of agents that enhance electric conductivity. Such modifications of the active material prior to electrode assembly have proven too time consuming, low yielding and expensive and, hence, none of these methods have found their way into commercial processes to date.

In this work, we present a departure from the current "blend-andbake" paradigm of electrode manufacture. In a one-pot process, we embed silicon nanoparticles (Si NPs) in a covalently-linked, porous, semi-conducting polymer matrix whose growth is initiated and templated by the current collector $(\mathrm{Cu})$ itself. The covalent bonds of the organic matrix contribute to a superior mechanical and chemical resistance of our electrode films. The overall $\pi$ conjugated backbone of the triazine-based graphdiyne ( $T z G)$ polymer enables the transport of electrons from the active material to the current collector. Since the polymerization is promoted by the reactive metal surface of the current collector, the resulting polymer/silicon composite (TzG/Si) adheres strongly to it. ${ }^{[7]}$ In summary, the covalent polymer matrix acts at the same time as (i) a strong binder, as (ii) an electrical conductor, and as (iii) a semi-permeable membrane that enables transport of ions and electrolyte but prevents the migration of homogeneously dispersed silicon nanoparticles even under harsh conditions. This facile method yields silicon-based anodes (TzG/Si@Cu) of superior performance that suffer little mechanical and electrochemical deterioration from the inherent volume expansion of silicon during lithiation-delithiation cycles and that drastically limit the detrimental loss of lithium and electrolyte at the solid electrolyte interface.

\section{Results and Discussion}

Electrodes of TzG/Si@Cu are prepared by dissolving the organic monomer 2,4,6-tris(4-ethynylphenyl)-1,3,5-triazine and dispersing Si NPs in pyridine in a $25 \%: 75 \%$ weight ratio, respectively. The reaction mixture is then transferred onto a copper foil (Figure 1a; Supplementary Information Section S1, Scheme $\mathrm{S} 1$ and S2). Residual $\mathrm{Cu}(\mathrm{II})$ and $\mathrm{Cu}(\mathrm{I})$ species on the untreated copper surface initiate the polymerization via a Glasertype oxidative coupling reaction. ${ }^{[8]}$ The polymerization is driven to completion and the pyridine is removed by evaporation. $\cdot{ }^{[7]}{ }^{13} \mathrm{C}$ cross-polarization-magic-angle-spinning (CP-MAS) solid-state NMR (Figure 1b) shows the characteristic signals of a triazinebased graphdiyne polymer; ${ }^{[7]}$ the triazine carbon at $\sim 170 \mathrm{ppm}$ and the diyne-bridges at $75-85 \mathrm{ppm}$. An additional signal seen at $\sim 30 \mathrm{ppm}$ is attributed to $\mathrm{O}_{2} \mathrm{SiMe}_{2}$ surface groups originating from the preparation of these commercially available Si NPs. This is corroborated by ${ }^{29} \mathrm{Si}$ single-pulse-magic-angle-spinning (SPMAS) solid-state NMR (Figure S1) and Fourier transform infrared (FT-IR) spectra (Figure S2). ${ }^{[9]}$ The Raman spectrum of TzG/Si@Cu (Figure 1C) shows stretching bands of diyne $\mathrm{C} \equiv \mathrm{C}$ at $2209 \mathrm{~cm}^{-1}$, of triazine $\mathrm{C}=\mathrm{N}$ at $1411 \mathrm{~cm}^{-1}$, of phenyl $\mathrm{C}=\mathrm{C}$ at $1604 \mathrm{~cm}^{-1}$, and of crystalline Si-Si bonds at $518 \mathrm{~cm}^{-1}$. [7, 10]

$\mathrm{X}$-ray photoelectron spectroscopy (XPS) performed on c-axis oriented layers of TzG/Si@Cu show all expected carbon environments in the $C$ 1s region that are observed for the neat
TzG polymer (Figure S3a). ${ }^{[7]}$ In addition, Si $2 p$ spectra show the presence of surface silicon oxide, $\mathrm{SiO}_{\mathrm{x}}(\sim 34 \%)$ and neat silicon $(66 \%)$ (Figure S3b), compared to as-received Si NPs that contain $21 \%$ of $\mathrm{SiO}_{x}$ and $79 \%$ of $\mathrm{Si}(0)$ environments (Figure S3d). ${ }^{[11]}$ In summary, spectroscopic analysis confirms the formation of a covalent, conjugated, triazine-based polymer network around chemically unchanged Si NPs.

For comparison, we have prepared three different types of electrode systems via the same one-pot method described above but with varying compositions to carefully test the effects of individual components: (i) growing a film of $\mathrm{TzG}$ on $\mathrm{Cu}$, we obtain TzG@Cu, (ii) growing TzG in the presence of Si NPs we get TzG/Si@Cu (in ration 25/75 wt\%), and (iii) TzG/Si/CB@Cu (in ratio 20/60/20 wt\%) is produced by growing TzG around Si NPs and a conventional, conductive additive, carbon black (CB) (Supplementary Information Section S1.5).

Scanning electron microscopy (SEM) images reveal the morphology of pristine TzG/Si@Cu electrodes. The material grown on the copper support adopts a porous, sponge-like, and homogeneous morphology as seen top-down (Figure 1e) and from cross-sections of the electrode film (Figure 1f). Crosssectional SEM imaging at lower magnifications shows films of $\mathrm{TzG} / \mathrm{Si}$ with a thickness of $\sim 25 \mu \mathrm{m}$ that adhere well to the $\mathrm{Cu}$ substrate with no apparent gaps (Figure S4). More detailed transmission electron microscopy (TEM) energy-filtered mapping on TzG/Si films shows a homogenous distribution of carbon and silicon on the nano-scale (Figure 1g). On the nanoscale, the electrode film consists of Si NPs homogenously embedded in an organic polymer matrix of TzG. Residual Cu nanoparticles can be seen within the polymer matrix that stem from the $T z G$ polymerization process (Figure $1 \mathrm{~h}$; a comparison of TEM images of TzG@Cu and of pristine Si NPs can be found in Figure S5)..7] Overall, individual Si NPs are enclosed by the conjugated, polymer and held cooperatively as a film on the current collector. We have shown previously that neat, unmodified triazine-based graphdiyne polymers are narrow-band gap semiconductors $\left(E_{\mathrm{g}, \text { elec }}=1.84 \mathrm{eV}\right.$ and conductivity of $\left.1.2 \mu \mathrm{S} \mathrm{cm}^{-1}\right)$ with moderate porosities $\left(\mathrm{N}_{2}\right.$ BET surface area of $124 \mathrm{~m}^{2} \mathrm{~g}^{-1}$ at $\left.77 \mathrm{~K}\right){ }^{\left[7,{ }^{12]} \text { Hence, }\right.}$ the composite $\mathrm{TzG} / \mathrm{Si}$ on $\mathrm{Cu}$ foil $(\mathrm{TzG} / \mathrm{Si} @ \mathrm{Cu}$ ) has a promising combination of chemical, electrical, and structural features for electrochemical storage.

In the following (Supplementary Information Section S2.1), we discuss the electric and electrochemical performance of TzGbased electrodes and the effects of the TzG polymer on the formation of the SEI. For the three electrode systems TzG/Si@Cu, TzG/Si/CB@Cu, and TzG@Cu (i) we compared the bulk conductivities of the unlithiated, "as-synthesized" electrodes (Figure S6), (ii) we recorded cyclic voltammetry (CV) curves (Figure S7), and (iii) we performed ex-situ XPS measurements probing the electrode surfaces to a depth of approx. $10 \mathrm{~nm}$ after a number of de-/lithiation cycles (Figure 2a; Figure S8, S9, S10 and S13; Table S1; details of the XPS spectra fitting method are described in Supplementary Information section S1.7). During the first lithiation of the TzG/Si@Cu electrode, the resulting composition of the film present a content with up to $43.9 \mathrm{~mol} \%$ of cumulative Li, F. and P elements (Table 1). Furthermore, after the first cycle of lithiation and delithiation, we observe that the initial Si $2 p$ signal of the pristine electrode decreases dramatically in intensity from $40.8 \mathrm{~mol} \%$ to value close to zero (Table 1). The 

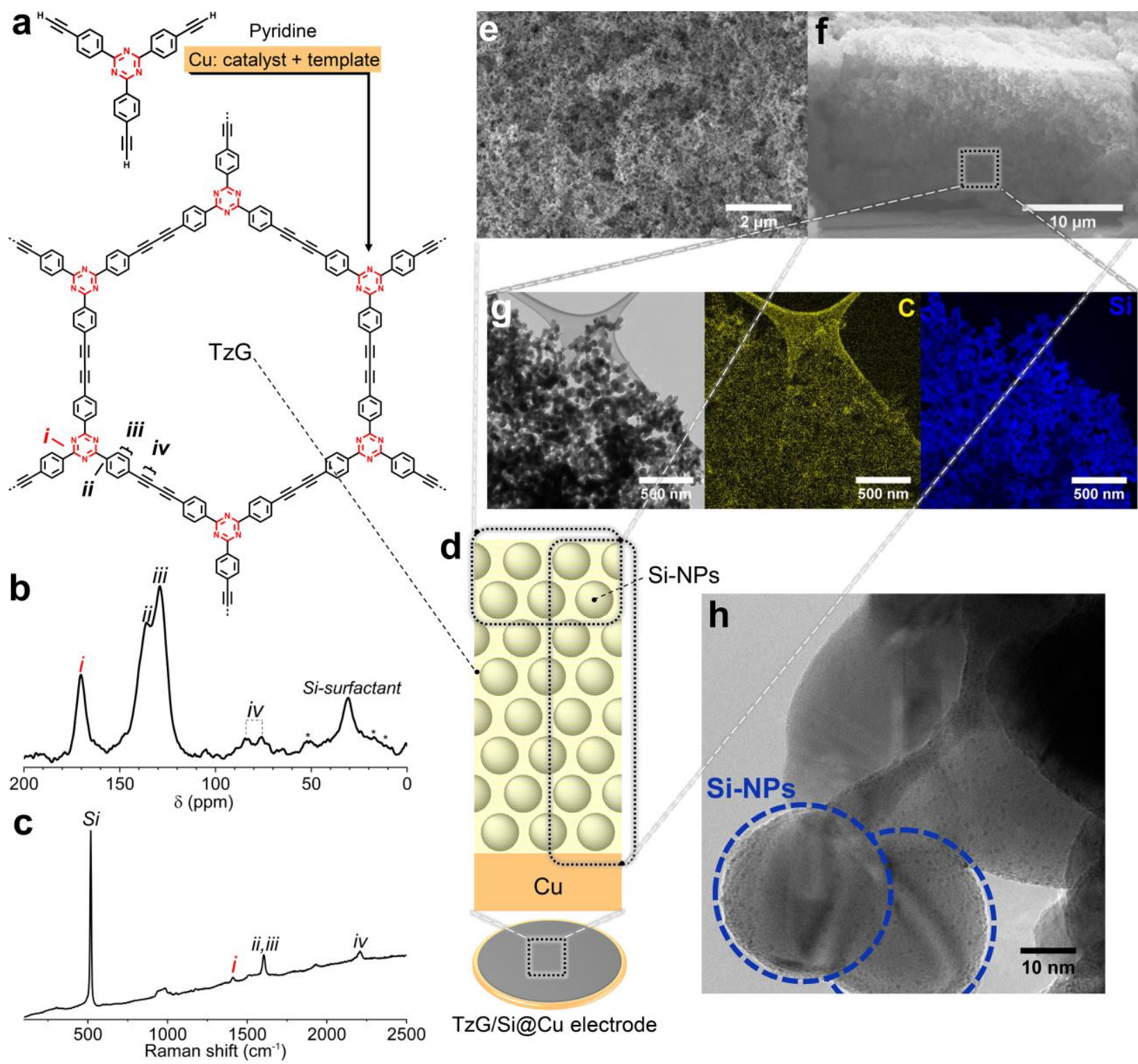

Si-NPs

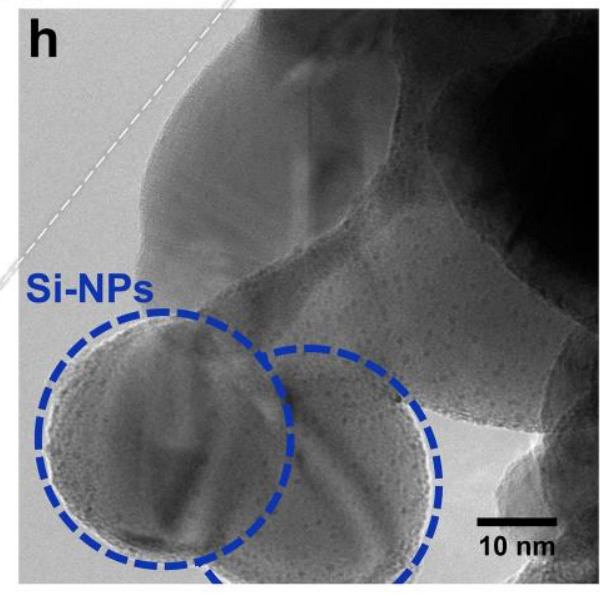

TzG/Si@Cu electrode

Figure 1. Micro- and macroscopic characterization of the chemical make-up of TzG/Si@Cu (TzG/Si=25/75 wt\%) electrodes. a, Synthetic pathway for the triazinebased graphdiyne (TzG) polymer. Spectroscopic characterization showing the corresponding $\mathbf{b},{ }^{13} \mathrm{C}$ cross-polarization-magic-angle-spinning (CP-MAS) solid-state NMR spectrum of the TzG/Si polymer composite, and c, the Raman spectrum with fluorescence background collected with a $532 \mathrm{~nm}$ laser. d, Schematic of the TzG/Si@Cu electrode. Scanning electron microscopy (SEM) images showing e, the top-view surface morphology and f, the cross section morphology of TzG/Si@Cu films. g, Low-resolution transmission electron microscopy (TEM) images and energy-filtered mapping images showing the homogenous distribution of carbon and silicon environments, and $\mathbf{h}$, high-resolution TEM images showing Si NPs fully enclosed by TzG polymer.

fitting of C 1s, F 1s and Li 1s spectra (Figure S10) indicate the formation of the SEI during the first lithiation, resulting in the variations of surface elemental concentration mentioned above. Note though that even after the 100th delithiation event, we detect a signal of $\mathrm{Si}(0)$ in the $\mathrm{Si} 2 \mathrm{p}$ region (Figure $2 \mathrm{a}$, iv, Table 1 ). This finding suggests that SEI formation is completed at an early stage, and that SEI thickness does not increase dramatically throughout cycling. ${ }^{[13]}$ XPS data from the $\mathrm{Si} 2 \mathrm{p}$ region of TzG/Si@Cu electrodes cycled at $\mathrm{C} / 8$ (Figure 2a) shows the emergence of new Si $2 p$ peaks corresponding to $\mathrm{Li}_{x} \mathrm{Si}, \mathrm{SiO}_{x} \mathrm{~F}_{y}$, and $\mathrm{Li}_{x} \mathrm{SiO}_{y}$ after the first lithiation event. ${ }^{[11]}$ The presence of $\mathrm{SiO}_{x} \mathrm{~F}_{y}$ results from the non-faradic reaction between the remaining $\mathrm{LiPF}_{6}$ and the $\mathrm{SiO}_{x}$ on the surface of the Si NPs, during the opening the cell for exsitu measurement. ${ }^{[11 \mathrm{a}]}$ The environments of $\mathrm{Li}_{x} \mathrm{Si}$ and $\mathrm{Li}_{x} \mathrm{SiO}_{y}$ originate from the electrochemical alloying reaction of silicon and $\mathrm{SiO}_{x}$ with the lithium ions in the electrolyte, as further corroborated by emerging signals in the $\mathrm{F} 1 \mathrm{~s}$ and $\mathrm{Li}$ 1s regions (Figure $\mathrm{S} 10 \mathrm{~b}$ and S10c)..$^{[11 b, 11 d]}$ Pristine and delithiated samples of TzG/Si@Cu all show peaks corresponding to $\mathrm{Si}(0)$ and $\mathrm{SiO}_{\mathrm{x}}$ indicating a fully reversible de-/lithiation process.

Ex-situ TEM and SEM imaging of TzG/Si@Cu electrodes at different cycling stages (Figure $2 b$ and $c$ ) reveal that prior to cycling, the TzG polymer acts as a binder that joins Si NPs into a porous TzG/Si composite (i). After the first lithiation event, 

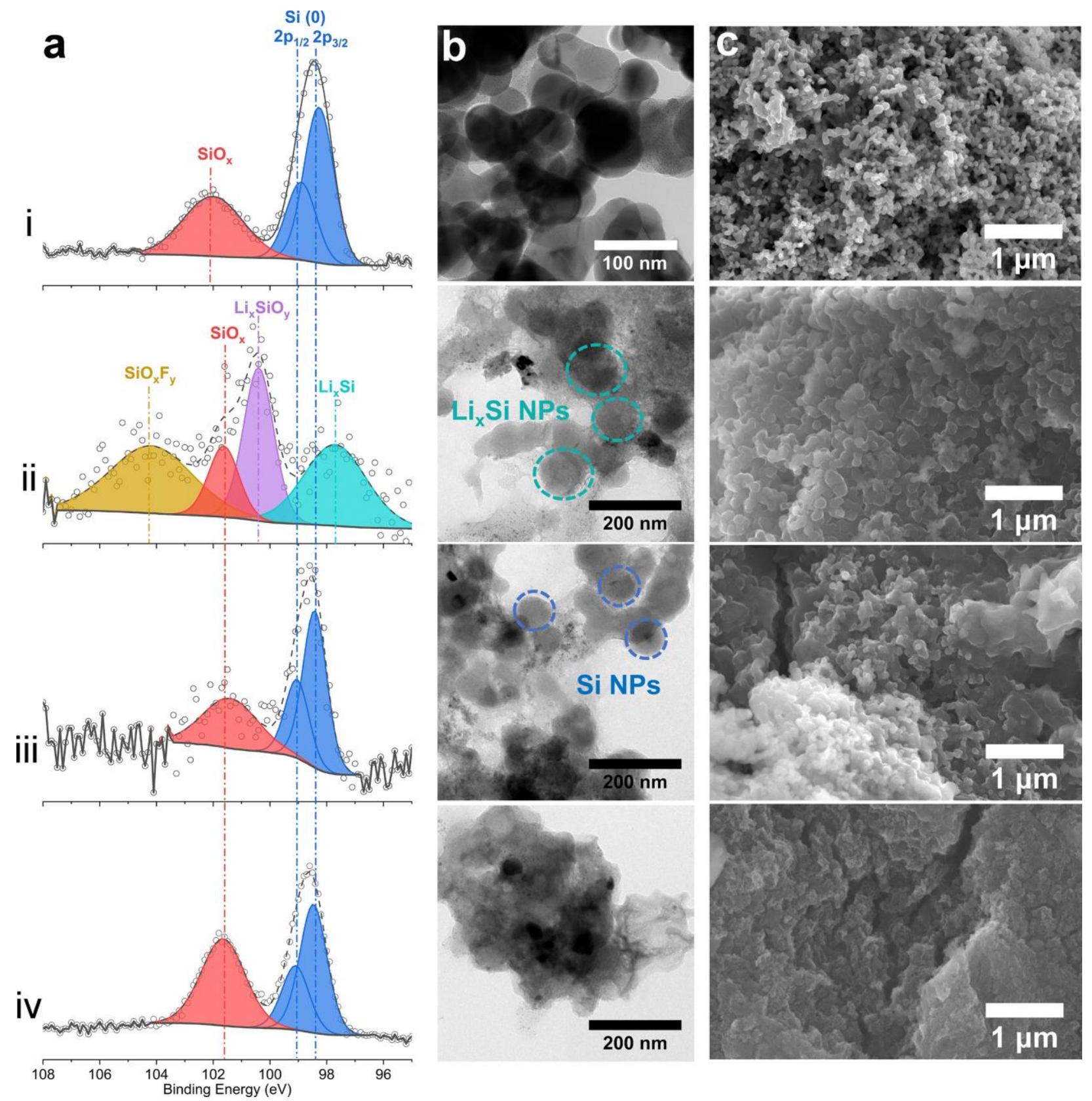

Figure 2. Characterization of TzG/Si@Cu electrodes using a, X-ray photoelectron spectroscopy (XPS) data from the Si 2p region, b, transmission electron microscopy (TEM), and c, scanning electron microscopy (SEM). Data is presented for TzG/Si@Cu electrodes in the pristine state (i), after the first lithiation (ii), after the first delithiation (iii) and after the $100^{\text {th }}$ delithiation (iv). Cycling was performed at a constant current of $0.32 \mathrm{~mA} \mathrm{~cm}{ }^{-2}$ (i.e. C/8 for the capacity of Si, within 0.01 $1.2 \mathrm{~V}$ vs. $\left.\mathrm{Li}^{-} \mathrm{Li}^{+}\right)$.

individual particles of increased size can be discerned that correspond to lithiated and volumetrically expanded domains of $\mathrm{Li}_{x} \mathrm{Si}$ all embedded in a fabric of polymer and SEI (ii). After the first delithiation, the size of the spherical Si domains decreases (iii), and after the $100^{\text {th }}$ delithiation, all that can be said is that the $\mathrm{TzG} / \mathrm{Si}$ composite shows no discernible large cracks or deformations (iv) (Figure S11 and S12).

A comprehensive discussion of these findings is presented in Supplementary Information section S2.2. For now, we conclude that: (i) the alloying reaction between lithium and Si NPs takes place unhindered, hence, the TzG polymer matrix allows lithium to diffuse, (ii) the conductivity enhancing additive in TzG/Si/CB@Cu has no positive effect on the performance of TzGbased electrodes, hence, the initial, modest conductivity of undoped TzG is no impediment for its use in electrodes; and (iii) the $\mathrm{TzG}$ polymer does not participate in the detrimental depletion of lithium or electrolyte on its own, and SEI formation occurs exclusively in the presence of $\mathrm{Si} \mathrm{NPs}$. It is particularly surprising that TzG-based electrodes perform as well as they do, given their low conductivity in the pristine, unlithiated state. While an in-depth study of the electric conductivity of lithiated TzG/Si@Cu is out of the scope of this work, XPS results for TzG@Cu suggest that the 

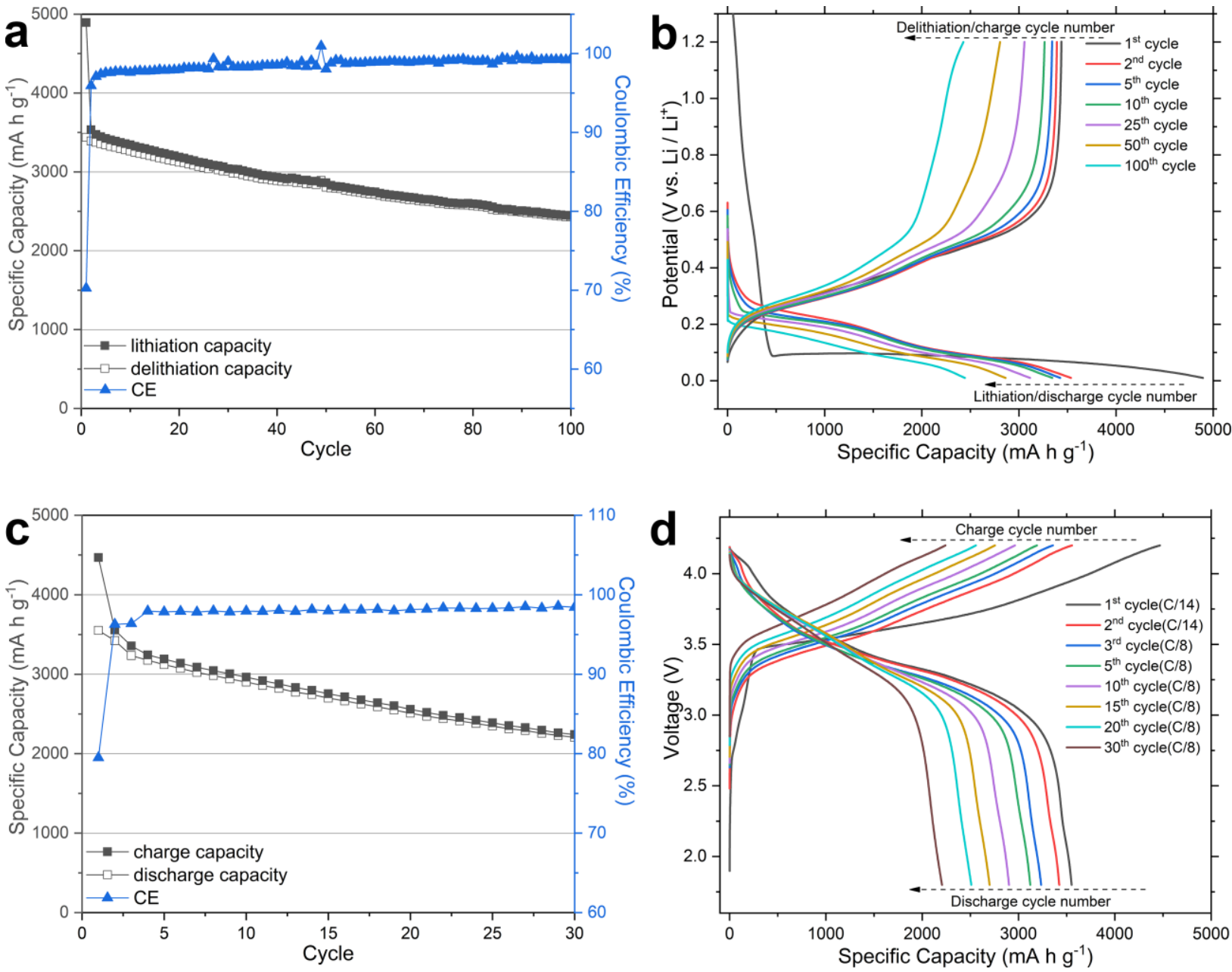

Figure 3. Half-cell performance data of the TzG/Si@Cu electrodes. Galvanostatic charge-discharge cycling with potential limitation (GCPL) uses constant current mode at $\mathrm{C} / 8$ within a voltage range of $0.01-1.2 \mathrm{~V}$ vs. Li/Li+ . a, Specific capacities normalized to Si mass and Coulombic efficiencies (CE) vs. cycle number. $\mathbf{b}$, Potential vs. specific capacity curves for consecutive de-/lithiation cycles. Full-cell performance data for an assembly consisting of a TzG/Si@Cu anode paired with a NCM811 cathode. The $\mathrm{n} / \mathrm{p}$ ratio is 0.94 (NCM811 loading of $6.502 \mathrm{mg} \mathrm{cm}^{-1}$ and Si loading of $0.291 \mathrm{mg} \mathrm{cm}^{-2}$ ). The full-cell was precycled at C/14 (based on the Si mass-loading) for two initial cycles, and at C/8 for all subsequent cycles, within a potential window of $4.2-1.8 \mathrm{~V}$. c, Specific capacities normalized to Si mass / CE vs. cycle number and $\mathbf{d}$, charge-discharge curves.

first lithiation cycle leads to $\mathrm{n}$-doping of the TzG polymer by $\mathrm{N}$ 1s signal shifting to higher binding energy (Figure S8), ${ }^{[14]}$ analogous to lithiated graphite, ${ }^{[15]}$ and, hence, to an increase of its conductivity above and beyond the effect achieved by the conducting additive in TzG/Si/CB@Cu.

We subjected the TzG/Si@Cu electrode to de-/lithiation cycling at $\mathrm{C} / 8$ in a half-cell set-up recording specific capacity (Figure 3 ). In the first lithiation cycle, we observe the formation of the SEI at a comparatively low CE value of $\sim 70 \%$, meaning that the surface of Si NPs is readily accessible for lithium and for the electrolyte via the pore channels of the TzG polymer. Similar Coulombic efficiencies have been reported for nanosized silicon particles with a large external surface area. Microsized silicon particles with a smaller ratio of exposed surfaces show higher initial CE values but suffer from limited discharge capacities and poor cycling performance. ${ }^{[16]}$ In the third cycle, the CE rises above $97 \%$ and steadily improves up to $99.5 \%$ which means that SEI formation is completed early on. The specific capacity observed during the first lithiation exceeds the theoretical value for fully lithiated silicon
(3579 $\mathrm{mA} \mathrm{h} \mathrm{g}^{-1}$ ) due to the formation of the SEl. ${ }^{[17]}$ Starting with the second cycle, no excess capacity is recorded and, hence, the newly formed SEI does not contribute to the measured discharge capacity. The recorded specific lithiation capacity in the second cycle is $\sim 3500 \mathrm{~mA} \mathrm{~h} \mathrm{~g}^{-1}$, and it remains at a record high in subsequent cycles (e.g. $\sim 3450 \mathrm{~mA} \mathrm{~h} \mathrm{~g}^{-1}$ in the fifth cycle). In comparison, the TzG/Si/CB@Cu electrode prepared together with carbon black does not perform better than the additive-free electrode (Figure S14). Taking the specific lithiation capacity in the second cycle as a baseline, TzG/Si@Cu retains $81.0 \%$ of its specific capacity after 50 cycles $\left(2862 \mathrm{~mA} \mathrm{~h} \mathrm{~g}^{-1}\right)$ and $69.1 \%$ after 100 cycles $\left(2443 \mathrm{~mA} \mathrm{~h} \mathrm{~g}^{-1}\right)$ Conversions of these values to areal and volumetric capacities can be found in Figure S15.

For comparison, we prepared two electrodes using conventional binders and CB additive, PVdF/Si/CB@Cu and PAA/Si/CB@Cu (Figure S16). ${ }^{[11 \mathrm{c}, 18]}$ These conventional formulations allow a total Si mass loading of up to $60 \%$ without compromising the performance of the electrode, compared to $75 \%$ of $\mathrm{Si}$ in the TzG/Si@Cu system. Overall, the specific capacity and retention 
Table 1. Relative molar concentrations of elements as determined by peak integration using X-ray photoelectron spectroscopy (XPS) data recorded at 1486.6 eV for TzG/Si@Cu electrodes at various stages of de-/lithiation cycled at a constant current of $0.32 \mathrm{~mA} \mathrm{~cm}^{-2}$ (i.e. C/8 for the capacity of $\mathrm{Si}$, within $0.01-1.2 \mathrm{~V}$ vs. Li/Li+). (Calculated based on XPS C 1s, Si 2p N 1s, O 1s, Li 1s, F 1s and P 2p spectra).

\begin{tabular}{lllllllll}
\hline Sample & Stage during cycling & $\mathrm{C}(\%)$ & $\mathrm{Si}(\%)$ & $\mathrm{N}(\%)$ & $\mathrm{O}(\%)$ & $\mathrm{Li}(\%)$ & $\mathrm{F}(\%)$ & $\mathrm{P}(\%)$ \\
\hline TzG/Si@Cu & pristine & 17.8 & 40.8 & 2.17 & 39.3 & 0.00 & 0.00 & 0.00 \\
TzG/Si@Cu & 1st lithiation & 23.0 & 1.90 & 0.65 & 28.2 & 28.1 & 15.8 & 2.36 \\
TzG/Si@Cu & 1st delithiation & 29.4 & 1.71 & 0.16 & 27.3 & 22.4 & 17.8 & 1.24 \\
TzG/Si@Cu & 100th delithiation & 44.5 & 0.84 & 0.00 & 32.0 & 15.9 & 6.31 & 0.44 \\
\hline
\end{tabular}

of TzG/Si@Cu anodes exceeds that of the best-performing multicomponent systems with and without conductivity-enhancing additives that are reported to date (Table S3). ${ }^{[19]}$ We tuned massloading of TzG/Si@Cu electrodes that lead to similar excellent performance (Supplementary information Table S2, Section S2.6, Figure S17-S19). For example, we were able to increase the Si mass-loading of the electrode beyond $1 \mathrm{mg} \mathrm{cm}^{-2}$ by a two rounds of polymerisation on top of a TzG/Si@Cu electrode in the presence of small quantities of $\mathrm{Cu}(\mathrm{OAc})_{2}$ as an additional source of $\mathrm{Cu}$ (II) species (Supplementary Information Section S1.6, Table S2). The obtained electrodes show a stable cycling performance and comparably high capacities as electrodes obtained in a onestep growth process (Figure S19).

TzG is a thermally stable polymer with a decomposition onset above $400{ }^{\circ} \mathrm{C}$ under air. ${ }^{[7]}$ Hence, we tested the performance of the TzG/Si@Cu half-cell after a heat treatment of $80^{\circ} \mathrm{C}$ for $6 \mathrm{~h}$, above temperatures experienced by $\mathrm{Li}$-ion batteries in some industrial and military settings. The overall performance of the TzG/Si@Cu half-cell after the thermal-stress test remains at $\sim 3000 \mathrm{~mA} \mathrm{~h} \mathrm{~g}^{-1}$ in the second cycle comparable to the performance of untreated electrodes. The difference in overall capacity and in capacity retention can be attributed to partial decomposition of $\mathrm{LiPF}_{6}$ during the extended heat treatment (Figure S20). As a proof of concept, we assembled a full cell using TzG/Si@Cu as the anode (Si areal mass-loading of $0.291 \mathrm{mg} \mathrm{cm}$ ${ }^{2}$, specific capacity $3579 \mathrm{~mA} \mathrm{~h} \mathrm{~g}^{-1}$ ) and the commercially available standard NCM811 as the cathode (areal mass-loading of $6.502 \mathrm{mg} \mathrm{cm}^{-2}$, specific capacity $\sim 170 \mathrm{~mA} \mathrm{~h} \mathrm{~g}^{-1}$ ) (Figure S21). The NCM811 cathode was selected over three commercial options (NCM532, NCM622, and NCA) as the one with the highest specific capacity and most stable cycling performance. Even though the specific capacities of our anode and the commercial standard NCM811 cathode are mismatched, the fullcell assembly shows an acceptable initial CE of $79 \%$ that stabilises at $>98 \%$ after the fourth cycle. Overall, the full-cell has a capacity of $3234 \mathrm{~mA} \mathrm{~h} \mathrm{~g}^{-1}$ in the third cycle and a capacity retention of $68.2 \%$ over 30 cycles (Figure $3 c$ and $3 d$, Supplementary information Section S2.7, Figure S22). We believe that full-cell assemblies with better coulombic efficiencies can be achieved using cathodes that (i) match the high capacity of our anode better, and (ii) have similar diffusion kinetics.

\section{Conclusion}

We present here a one-pot synthetic protocol that yields highperformance silicon-lithium anodes within one hour of reaction time. These anodes consist of silicon nanoparticles that are fully encapsulated by a semi-conducting, porous triazine-based graphdiyne ( $T z G)$ polymer that grows directly on the $\mathrm{Cu}$ current collector. $\mathrm{Cu}$ foil plays three roles in this paradigm-changing method of anode fabrication: it acts (i) as a source of Cu species for a Glaser-type oxidative coupling polymerization, (ii) as templating substrate for the polymer film, and (iii) as the current collector of the electrode. The porous, semi-conducting TzG polymer acts (i) as a strong, flexible binder that envelops Si NPs with a matrix of covalent bonds that can sustain the dramatic volume changes of silicon in repeated de-/lithiation cycles and prevents detrimental abrasion and reformation of the solid electrolyte interface, (ii) as a facilitator of charge transport along its $\pi$-conjugated polymer backbone, and (iii) as a medium for mass transport of lithium ions and electrolyte through its microporous channels. The resulting anodes achieve stable electrochemical cycling performance and an extraordinarily high capacity close to the theoretical limit of electrochemical storage using silicon. The reported process uses raw materials and methods common in industrial electrode manufacture and can be transferred and scaled up with ease. Half-cell electrode assemblies in the off-state retain key performance parameters even after thermal stress, and full-cell cycling tests using commercial cathodes demonstrate the viability of this technology in commercial applications.

\section{Acknowledgements}

We thank Dr. Martin Dračínský for solid-state NMR measurements, Dr. Petr Formánek for TEM imaging and mapping, Prof. Dr. Jürgen P. Rabe for access to Raman spectroscopy. J.H. thanks Dr. Mathias Trunk for providing monomer materials, Weimiao Wang for Raman spectra discussion and analysis. M.J.B. thanks the European Research Council (ERC) for funding under the Starting Grant Scheme (BEGMAT-678462) and the Proof of Concept Grant Scheme (LiAnMat-957534).

Keywords: graphdiyne $\cdot$ glaser-coupling $\bullet$ Li-ion battery $\bullet$ silicon anode $\cdot$ one-pot

[1] R. Schmuch, R. Wagner, G. Hörpel, T. Placke, M. Winter, Nat. Energy 2018, 3, 267.

[2] J. Xu, Y. Dou, Z. Wei, J. Ma, Y. Deng, Y. Li, H. Liu, S. Dou, Adv. Sci. 2017, 4, 1700146 .

[3] T.-w. Kwon, J. W. Choi, A. Coskun, Chem. Soc. Rev. 2018, 47, 2145 2164 
[4] aG. G. Eshetu, E. Figgemeier, ChemSusChem 2019, 12, 2515-2539; bP. Cao, Y. Pan, S. Gao, F. Sun, H. Yang, Chem. Eur. J. 2019, 25, 10976 10994; cP. Li, G. Zhao, X. Zheng, X. Xu, C. Yao, W. Sun, S. X. Dou, Energy Storage Mater. 2018, 15, 422-446; dK. Feng, M. Li, W. Liu, A. G. Kashkooli, X. Xiao, M. Cai, Z. Chen, Small 2018, 14, 1702737.

[5] N. Liu, H. Wu, M. T. McDowell, Y. Yao, C. Wang, Y. Cui, Nano Lett. 2012 12, 3315-3321.

[6] H. T. Nguyen, M. R. Zamfir, L. D. Duong, Y. H. Lee, P. Bondavalli, D. Pribat, J. Mater. Chem. 2012, 22, 24618-24626.

[7] D. Schwarz, Y. Noda, J. Klouda, K. Schwarzová - Pecková, J. Tarábek, J. Rybáček, J. Janoušek, F. Simon, M. V. Opanasenko, J. J. A. M. Čejka, Adv. Mater. 2017, 29, 1703399

[8] aH. Möhwald, V. Bliznyuk, S. Kirstein, Synth. Met. 1993, 61, 91-96; bY Li, L. Xu, H. Liu, Y. Li, Chem. Soc. Rev. 2014, 43, 2572-2586; cV. S Vyas, F. Haase, L. Stegbauer, G. Savasci, F. Podjaski, C. Ochsenfeld, B. V. Lotsch, Nat. Commun. 2015, 6, 1-9.

[9] aF. Uhlig, H. C. Marsmann, Gelest Catalog 2008, 208-222; bC.-M. Park, W. Choi, Y. Hwa, J.-H. Kim, G. Jeong, H.-J. Sohn, J. Mater. Chem. 2010 20, 4854-4860; cJ. Mijatovic, W. H. Binder, H. Gruber, Microchim. Acta 2000, 133, 175-181.

[10] aP. Larkin, M. Makowski, N. Colthup, Spectrochim. Acta - A: Mol. Biomol. Spectrosc. 1999, 55, 1011-1020; bH. Li, X. Huang, L. Chen, G. Zhou, Z Zhang, D. Yu, Y. J. Mo, N. Pei, Solid State Ion. 2000, 135, 181-191.

[11] aB. Philippe, R. m. Dedryvère, M. Gorgoi, H. k. Rensmo, D. Gonbeau, K. Edström, Chem. Mater. 2013, 25, 394-404; bE. Radvanyi, E. De Vito, W Porcher, S. J. S. Larbi, J. Anal. At. Spectrom. 2014, 29, 1120-1131; cC C. Nguyen, T. Yoon, D. M. Seo, P. Guduru, B. L. Lucht, ACS Appl. Mater Interfaces 2016, 8, 12211-12220; dG. Ferraresi, L. Czornomaz, C. Villevieille, P. Novák, M. El Kazzi, ACS Appl. Mater. Interfaces 2016, 8 29791-29798.

[12] D. Schwarz, A. Acharjya, A. Ichangi, Y. S. Kochergin, P. Lyu, M. V. Opanasenko, J. Tarábek, J. Vacek Chocholoušová, J. Vacek, J. Schmidt, ChemSusChem 2019, 12, 194-199.

[13] aJ. Xu, Theses and Dissertations--Chemical and Materials Engineering. 68. 2016, https://uknowledge.uky.edu/cme etds/68; bB. Koo, H. Kim, Y Cho, K. T. Lee, N. S. Choi, J. Cho, Angew. Chem. Int. Ed. 2012, 51, 8762-8767.

[14] aL. K. Putri, B.-J. Ng, W.-J. Ong, H. W. Lee, W. S. Chang, S.-P. Chai, J. Mater. Chem. A 2018, 6, 3181-3194; bK. Sakaushi, G. Nickerl, F. M. Wisser, D. Nishio - Hamane, E. Hosono, H. Zhou, S. Kaskel, J. Eckert, Angew. Chem. Int. Ed. 2012, 51, 7850-7854; cH. Sezen, S. Suzer, J. Chem. Phys. 2011, 14, 141102

[15] aG. Wertheim, P. T. M. Van Attekum, S. Basu, Solid State Commun 1980, 33, 1127-1130; bA. M. Andersson, A. Henningson, H. Siegbahn, U. Jansson, K. Edström, J. Power Sources 2003, 119, 522-527.

[16] W.-F. Ren, J.-B. Le, J.-T. Li, Y.-Y. Hu, S.-Y. Pan, L. Deng, Y. Zhou, L. Huang, S.-G. Sun, ACS Appl. Mater. Interfaces 2021, 13, 639-649.

[17] aM. Obrovac, L. Christensen, Electrochem. Solid-State Lett. 2004, 7, A93-A96; bl. Kovalenko, B. Zdyrko, A. Magasinski, B. Hertzberg, Z. Milicev, R. Burtovyy, I. Luzinov, G. Yushin, Science 2011, 334, 75-79; cA. Urbanski, A. Omar, J. Guo, A. Janke, U. Reuter, M. Malanin, F. Schmidt, D. Jehnichen, M. Holzschuh, F. Simon, J. Electrochem. Soc. 2019, 166 A5275-A5286.

[18] Z. Karkar, D. Guyomard, L. Roué, B. Lestriez, Electrochim. Acta 2017, 258, 453-466.

[19] aZ. Chen, C. Wang, J. Lopez, Z. Lu, Y. Cui, Z. Bao, Adv. Energy Mater. 2015, 5, 1401826; bS. Choi, T.-w. Kwon, A. Coskun, J. W. Choi, Science 2017, 357, 279-283; cT. M. Higgins, S.-H. Park, P. J. King, C. Zhang, N McEvoy, N. C. Berner, D. Daly, A. Shmeliov, U. Khan, G. Duesberg, ACS Nano 2016, 10, 3702-3713; dY. K. Jeong, T.-w. Kwon, I. Lee, T.-S. Kim, A. Coskun, J. W. Choi, Energy Environ. Sci. 2015, 8, 1224-1230; eG. Liu, S. Xun, N. Vukmirovic, X. Song, P. Olalde - Velasco, H. Zheng, V. S Battaglia, L. Wang, W. Yang, Adv. Mater. 2011, 23, 4679-4683; fJ. Song M. Zhou, R. Yi, T. Xu, M. L. Gordin, D. Tang, Z. Yu, M. Regula, D. Wang, Adv. Funct. Mater. 2014, 24, 5904-5910; gC. Wang, H. Wu, Z. Chen, M T. McDowell, Y. Cui, Z. Bao, Nat. Chem. 2013, 5, 1042; hH. Wu, G. Yu, L. Pan, N. Liu, M. T. McDowell, Z. Bao, Y. J. N. c. Cui, Nat. Commun. 2013, 4, 1943; iW. Zeng, L. Wang, X. Peng, T. Liu, Y. Jiang, F. Qin, L. Hu, P. K. Chu, K. Huo, Y. Zhou, Adv. Energy Mater. 2018, 8, 1702314;
jG. Zhang, Y. Yang, Y. Chen, J. Huang, T. Zhang, H. Zeng, C. Wang, G. Liu, Y. Deng, Small 2018, 14, 1801189. 


\section{Entry for the Table of Contents}

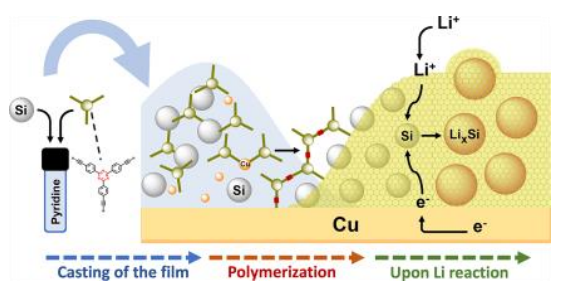

We replace the conventional components in silicon-lithium anodes by growing a triazine-based graphdiyne polymer network around silicon nanoparticles directly on the current collector, a copper sheet. The porous, semi-conducting polymer binds silicon to the current collector, conducts electrons, and enables diffusion of electrolyte and Li-ions. The resulting anode has a capacity at the theoretical limit of fully lithiated silicon. 